\title{
Noninvasive Acoustic Respiration Rate Monitoring
}

\author{
Kentaro Ouchi* \\ Department of Dental Anesthesiology, Field of Maxillofacial Diagnostic and Surgical Sciences \\ Faculty of Dental Science, Kyushu University Graduate School, Japan
}

Received: February 23, 2016; Accepted: April 12, 2016, Published: May 24, 2016

*Corresponding author: Kentaro Ouchi, Department of Dental Anesthesiology, Field of Maxillofacial Diagnostic and Surgical Sciences, Faculty of Dental Science, Kyushu University Graduate School, 3-1-1 Maidashi, Higashi-ku, Fukuoka 812-8582, Japan, Tel: +81-92-641-1151; Fax: +81-92642-6481; Email: ken2006anes@yahoo.co.jp

\section{Abstract}

A new acoustic method for Respiratory Rate Monitoring ( $\mathrm{RRa}^{\circledR}$ ) has shown good reliability when used in patients without tracheal intubation. The objective of this review is to assess the usefulness of acoustic respiratory rate monitoring. In contrast to facemask capnometry, the acoustic monitor was well tolerated; contrary, capnometry was not well tolerated, because several patients removed the facemask. In intravenous general anesthesia, the acoustic monitor is useful for continuous monitoring of respiratory rate in spontaneously breathing patients. In pediatric patients, acoustic monitor showed good agreement and similar accuracy and precision between capnometry but was better tolerated.

In conclusion, the acoustic respiratory monitoring is useful for non-intubated patients.
\end{abstract}

\section{Introduction}

Several respiratory rate monitoring techniques are available. A new acoustic method for respiratory rate monitoring (RRa ${ }^{\circledR}$, Masimo Corp.) has shown good reliability when used in patients without tracheal intubation in dental operating room [1], postanesthesia care units [2,3], intensive care units [4], and emergency units [5]. Because there is no 'gold standard' for measurement of respiration rate, these reports were compared acoustic monitor with widely used methods (capnography or thoracic impedance pneumography). One such technique, capnography, is often used because of its ability to detect cessation of breathing before there is a decrease in pulse oxygen saturation. Capnography requires the use of a sampling line connected to an oral or nasal mask, and since the facemask for capnometry was removed many times, its use is limited [5]. We systematically searched Medline via PubMed using the keywords "acoustic respiratory rate monitoring". The electronic search was performed in January 2016. The publicized period was limited in 2012 or later. Case reports and original article irrelevant to $\mathrm{RRa}^{\circledR}$ were excluded.

The objective of this review is to assess the usefulness of acoustic respiratory rate monitoring. And, I have reviewed these reports in order to give some general recommendations.

\section{System of device}

A non-invasive respiratory monitoring device, using an adhesive sensor with an integrated acoustic transducer positioned on the patient's throat, has been recently introduced. This device analyses respiratory vibrations to detect inspiratory and expiratory flow. The sensor has been united with adhesive tape and acoustic transducer, in manufacture (Figure 1). Acoustic sensor was placed on the patient's throat, on the side of the larynx and above the thyroid cartilage. And acoustic sensor was connected to a specific device (Radical- $7^{\circledR}$ Pulse Oximeter, Masimo Corp.). The acoustic signal is then converted to continuous, numeric values of respiration rate.

The sensor allows real time analysis of the vibrations emanating from the patient's larynx and enables isolation of the respiratory sounds via analysis of the trace obtained through various filters. In this mechanism, the device seems to refer to the information from the pulse oximetry of the finger. The noise is removed by collating it with breathing pattern information from the pulse oximetry. However, the details of the mechanism are veiled in trade secrets. The acoustic signal is then converted to a numerical value, allowing a continuous display of the respiratory rate $(/ \mathrm{min})$

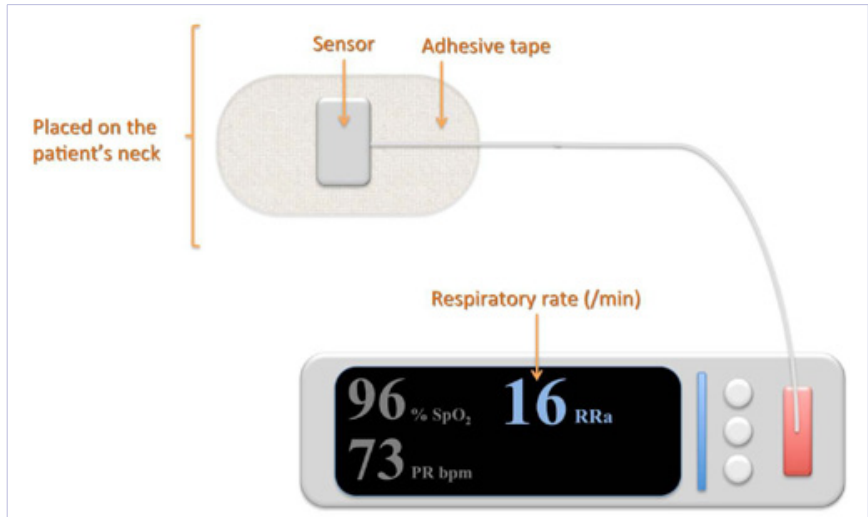

Figure 1: 


\section{The use under various situations}

\section{For post-anesthesia care}

Previous study reported that adult patients admitted to the post-anesthesia care unit (PACU) after extubation after general anesthesia were studied [2]. In this report, 42 patients with age of mean 54 years were enrolled. It is reported that continuous assessment of respiration rate with an acoustic monitor correlated well with capnometry. There were fewer errors of acoustic sensor than capnometry. The acoustic monitor was well tolerated; contrary, capnometry was not well tolerated, because eight patients removed the facemask.

Another previous study reported that adult patients admitted to PACU after extubation after general anesthesia were studied [3]. In this report, 33 patients with age of mean 45 years were enrolled. It is reported that, the acoustic monitor was statistically more accurate and more precise than the capnometry, but differences in performance were modest.

In comparison between capnography, the acoustic monitor is useful for continuous monitoring of respiratory rate in spontaneously breathing subjects for post-anesthesia care. Differences in performance were modest, unless removed the facemask.

\section{For emergency ward}

Previous study reported that adult patients admitted to emergency ward after extubation after drug or alcoholic poisoning were studied [5]. In this report, 30 patients with age of mean 37 years, hospitalized at the emergency department for drug or alcoholic poisoning, were enrolled. The acoustic monitor seems more accurate than thoracic impedance and better tolerated than facemask capnometry. Moreover, this report reported as follows. In adhesive tape with acoustic sensor, no allergic phenomenon was observed during the study. The acoustic sensor was repositioned in 3 cases, because the sensor got unstuck. The causes were agitation in 2 cases and substantial hairiness in 1 case. The electrodes for impedance measurement repositioned in 2 cases, because the sensor got unstuck. The facemask for capnometry was removed many times by 11 patients, so that emergency staff had to intervene and reposition them.

In comparison between thoracic impedance and capnography, the acoustic monitor is useful for continuous monitoring of respiratory rate in spontaneously breathing subjects for emergency ward.

\section{For general anesthesia with airway management}

Under airway management such as intubation, pneumotachograph with anesthesia machine and capnometry are useful for respiratory monitoring. However, the study using acoustic monitor in the operating room under general anesthesia with a laryngeal mask airway was reported [6]. In this report, 53 patients undergoing urologic procedures in the operating room under general anesthesia with a laryngeal mask airway, spontaneous ventilation and no muscle relaxation, were enrolled. Patients were monitored for respiration rate with acoustic monitor and in-circuit pneumotachograph. Instantaneous respiratory rates were obtained from the pneumotachograph. Acoustic monitor demonstrated a median delay of 45 seconds to detect a $1(/ \mathrm{min})$ change in instantaneous respiratory rates. And, acoustic monitor detects changes in respiratory rate, demonstrates minimal bias.

In comparison between normality widely used methods under airway management (capnography and pneumotachograph), normality widely used methods is useful for continuous monitoring of respiratory rate for general anesthesia with airway management, because acoustic monitor demonstrated delay to detect change in respiratory rates.

\section{For sedation for gastrointestinal endoscopy procedures}

Previous study reported that adult patients under propofolbased sedation for upper gastrointestinal endoscopy were studied [7]. In this report, 101 patients with age of mean 59 years undergoing upper gastrointestinal endoscopy under sedation without airway management such as tracheal intubation were enrolled. Patients were monitored for respiration rate with acoustic monitor, capnometry and impedance pneumography and values were compared to the manual counting of breaths by observation of chest wall movements. Acoustic monitor was found to be accurate for assessment of respiration rate and to have similar or better sensitivity and specificity for detection of apnea compared to capnometry and impedance pneumography in upper gastrointestinal endoscopy.

In comparison between thoracic impedance or capnography, the acoustic monitor is useful for detection of apnea in spontaneously breathing subjects for sedation during gastrointestinal endoscopy procedure.

\section{For intravenous anesthesia without tracheal intubation}

Previous study reported that adult patients with dental anxiety undergoing dental treatment under intravenous anesthesia without tracheal intubation were studied [1]. In this report, 11 patients with age of mean 38 years were enrolled. Respiratory rate was recorded every 30 seconds using the acoustic method and capnometry, and detect ability of respiratory rate was investigated for both methods. A total of 1953 data points of respiratory rate were obtained from the start to end of anesthesia. The number of detected points by the acoustic method $(1884,96.5 \%)$ was significantly higher than that by capnometry $(1682,86.1 \%)$.

In comparison between capnometry, the acoustic monitor is useful for continuous monitoring of respiratory rate in spontaneously breathing patients undergoing dental procedures under intravenous general anesthesia.

\section{For children}

Previous study reported that pediatric patients admitted to PACU after extubation after general anesthesia were studied [8]. In this report, 57 patients with age of mean 7years (range: 
1.2 - 15) were enrolled. The acoustic sensor was applied to the patient's neck according to the directions for use. Respiration rate data were recorded from acoustic monitor and capnometry. Patients of $97.5 \%$ demonstrated good tolerance of the acoustic monitor, where as patients of $62.5 \%$ demonstrated good tolerance of the capnometry. Acoustic monitor showed good agreement and similar accuracy and precision but was better tolerated in postsurgical pediatric patients.

In comparison between capnometry, the acoustic monitor is useful for continuous monitoring of respiratory rate in spontaneously breathing patients for postsurgical pediatric patients.

\section{False alarm}

Previous study that the use of acoustic respiratory monitoring in children receiving opioid infusions on a postsurgical ward was evaluated and false alarm was assessed was reported [9].This report indicated that, episodes of desaturation were observed in 9 patients, but the use of acoustic respiratory monitoring did not improve the detection of respiratory depression. And this reported that acoustic monitor resulted in an unacceptably high false alarm rate. Development and improvement of alarm system may be needed.

\section{Discussion}

In comparison between capnography, the acoustic monitor is useful for continuous monitoring of respiratory rate in spontaneously breathing subjects. Because capnometry is not tolerated when the sampling line was removed. It occurs when patients removed the facemask $[2,5]$. And it occurs at mouth breathing, under respiratory monitoring by capnography coupled with a nasal cannula [7]. In comparison between normality widely used methods (capnography and pneumotachograph) under airway management, normality widely used methods is useful than acoustic monitor for continuous monitoring of respiratory rate for general anesthesia with airway management. Because demonstrated delay to detect change in respiratory rates [6]. Therefore the acoustic respiratory monitoring is useful for nonintubated patients.

\section{Conclusion}

The acoustic respiratory monitoring is useful for nonintubated patients.

\section{References}

1. Ouchi K, Fujiwara S, Sugiyama K. Acoustic method respiratory rate monitoring is useful in patients under intravenous anesthesia. J Clin Monit Comput. 2016.

2. Mimoz 0, Benard T, Gaucher A, Frasca D, Debaene B. Accuracy of respiratory rate monitoring using a non-invasive acoustic method after general anaesthesia. Br J Anaesth. 2012;108(5):872-875.

3. Ramsay MA, Usman M, Lagow E, Mendoza M, Untalan E, De Vol E. The accuracy, precision and reliability of measuring ventilatory rate and detecting ventilatory pause by rainbow acoustic monitoring and capnometry. Anesth Analg. 2013;117(1):69-75.doi: 10.1213/ ANE.0b013e318290c798.

4. Autet LM, Frasca D, Pinsard M, Cancel A, Rousseau L, Debaene B, et al. Evaluation of acoustic respiration rate monitoring after extubation in intensive care unit patients. Br J Anaesth. 2014;113(1):195-197.

5. Guechi Y, Pichot A, Frasca D, Rayeh-Pelardy F, Lardeur JY, Mimoz O. Assessment of noninvasive acoustic respiration rate monitoring in patients admitted to an Emergency Department for drug or alcoholic poisoning. J Clin Monit Comput. 2015. 2015;29(6):721-726.

6. Atkins JH, Mandel JE. Performance of Masimo rainbow acoustic monitoring for tracking changing respiratory rates under laryngeal mask airway general anesthesia for surgical procedures in the operating room: a prospective observational study. Anesth Analg. 2014;119(6):1307-1314.

7. Goudra BG, Penugonda LC, Speck RM, Sinha JC. Comparison of acoustic respiration rate, impedance pneumography and capnometry monitors for respiration rate accuracy and apnea detection during GI endoscopy anesthesia. Open J Anesthesiol. 2013;3:74-79.

8. Patino M, Redford DT, Quigley TW, Mahmoud M, Kurth CD, Szmuk P. Accuracy of acoustic respiration rate monitoring in pediatric patients. Paediatr Anaesth. 2013;23(12):1166-1673.

9. Gorges M, West NC, Christopher NA, Koch JL, Brodie SM, Lowlaavar N, et al. An Ethnographic Observational Study to Evaluate and Optimize the Use of Respiratory Acoustic Monitoring in Children Receiving Postoperative Opioid Infusions. Anesth Analg. 2016;122(4):11321340. 